\title{
Contributions of Indirect Pathways to Visual Response Properties in Macaque Middle Temporal Area MT
}

\author{
Carlos R. Ponce, ${ }^{1,2}$ J. Nicholas Hunter, ${ }^{2}$ Christopher C. Pack, ${ }^{3}$ Stephen G. Lomber, ${ }^{4}$ and Richard T. Born ${ }^{2}$ \\ ${ }^{1}$ Harvard-Massachusetts Institute of Technology, Division of Health Sciences and Technology, and ${ }^{2}$ Department of Neurobiology, Harvard Medical School, \\ Massachusetts 02115, ${ }^{3}$ Montréal Neurological Institute, Montréal, Quebec H3A 2B4, Canada, and ${ }^{4}$ Department of Physiology and Pharmacology, The \\ University of Western Ontario, London, Ontario N6A 5C1, Canada
}

The primate visual cortex exhibits a remarkable degree of interconnectivity. Each visual area receives an average of 10 to 15 inputs, many of them from cortical areas with overlapping, but not identical, functional properties. In this study, we assessed the functional significance of this anatomical parallelism to the middle temporal area (MT) of the macaque visual cortex. MT receives major feedforward inputs from areas V1, V2, and V3, but little is known about the properties of each of these pathways. We previously demonstrated that reversible inactivation of $\mathrm{V} 2$ and $\mathrm{V} 3$ causes a disproportionate degradation of tuning for binocular disparity of MT neurons, relative to direction tuning (Ponce et al., 2008). Here we show that MT neurons continued to encode speed and size information during V2/3 inactivation; however, many became significantly less responsive to fast speeds and others showed a modest decrease in surround suppression. These changes resemble previously reported effects of reducing stimulus contrast (Pack et al., 2005; Krekelberg et al., 2006), but we show here that they differ in their temporal dynamics. We find no evidence that the indirect pathways selectively target different functional regions within MT. Overall, our findings suggest that the indirect pathways to MT primarily convey modality-specific information on binocular disparity, but that they also contribute to the processing of stimuli moving at fast speeds.

\section{Introduction}

The primate visual cortex consists of a large number of relatively discrete visual areas that are richly interconnected in a hierarchical arrangement (Felleman and Van Essen, 1991). One feature of this hierarchy is that any given visual area receives inputs from many other areas. What is the functional role of such convergence? One possibility is that different inputs may be highly redundant and serve primarily to increase the robustness of neural computations. A second is that different areas may provide qualitatively different information pertaining to, for example, motion or depth, which is selectively combined to solve particular perceptual problems (Van Essen et al., 1992). A third possibility is that different input streams are not combined at the single neuron level, but rather synapse in interdigitating sets of columns within the recipient area (Yabuta et al., 2001).

We investigated these possibilities by reversibly inactivating a subset of the inputs to the middle temporal visual area (MT). MT receives direct inputs from striate cortex $(\mathrm{V} 1)$, areas $\mathrm{V} 2$ and $\mathrm{V} 3$, as well as other cortical and subcortical regions (Zeki, 1969; Maunsell and Van Essen, 1983; Standage and Benevento, 1983). Because V2

Received 0ct. 12, 2010; revised Jan. 11, 2011; accepted Jan. 16, 2011.

C.C.P. was supported by a grant from the Canadian Institutes of Health Research (MOP-79352). C.R.P. was supported by a grant from the National Institute of Neurological Disorders and Stroke (F31NS052926). S.G.L. was supported by a grant from the Natural Sciences and Engineering Research Council of Canada (327442). R.T.B. was supported by National Eye Institute Grant R01 EY11379, a Vision Core Grant (EY12196), and a gift from R. Brandon Fradd. We are grateful to Phillip Hendrickson and Andrew Zaharia for their excellent technical assistance.

Correspondence should be addressed to Carlos R. Ponce, Harvard-MIT Division of Health Sciences and Technology, Harvard Medical School, 260 Longwood Avenue, Boston, MA 02115. E-mail: crponce@gmail.com.

DOI:10.1523/JNEUROSCI.5362-10.2011

Copyright $\odot 2011$ the authors $\quad 0270-6474 / 11 / 313894-10 \$ 15.00 / 0$ and V3 obtain their primary inputs from V1, MT is both a direct and an indirect target of V1. Previous findings suggest that these different projections serve different roles in visual processing; for example, there are morphological differences in the neuronal subpopulations in V1 that give rise to each pathway (Yabuta et al., 2001; Nassi and Callaway, 2006) in the laminar patterns of connectivity (Anderson and Martin, 2002), in the morphology of axons and axon terminals (Rockland, 1995; Anderson and Martin, 2002), and in average receptive field size (Gattass et al., 1981).

There are also differences at a functional level. V1 neurons contributing to the direct pathway are highly selective for direction of motion, respond to a broad range of temporal and spatial frequencies, and possess high-contrast sensitivity (Movshon and Newsome, 1996). MT-projecting neurons in V2 have not been individually characterized; however, the $\mathrm{V} 2$ regions that project to MT have a high proportion of binocular-disparity selective neurons and a relatively low incidence of direction selectivity (DeYoe and Essen, 1985; Hubel and Livingstone, 1987; Peterhans and von der Heydt, 1993).

Consistent with this, we previously showed that reversible inactivation of $\mathrm{V} 2$ and $\mathrm{V} 3$ caused a disproportionate degradation in the tuning of MT neurons for binocular disparity without significantly affecting their direction tuning (Ponce et al., 2008). In this article, we present data that further delineates the roles of inputs from $\mathrm{V} 2$ and $\mathrm{V} 3$ in the analysis of speed and size by MT neurons. We found that V2/V3 inactivation leads to reductions in the preferred speeds of many MT neurons. In general, these were modest effects compared with changes in binocular disparity, but they were consistent with the notion that the larger receptive fields in V2 and V3 can accommodate larger spatial displacements and 
thus endow MT neurons with the ability to respond to faster speeds. Additional experiments using smooth pursuit eye movements revealed that this effect can also be observed at the behavioral level.

\section{Materials and Methods}

\section{Behavioral tasks}

Six male macaques $(7-10 \mathrm{~kg}$ ) were trained to sit in custom chairs (Crist Instrument Co.) and perform a simple fixation task in which they were required to foveate a fixation target $\left(0.7^{\circ}\right.$ diameter $)$ within a fixation window of $2-3^{\circ}$ to obtain a juice reward every $2-3 \mathrm{~s}$. The fixation target was usually placed at the center of the monitor but occasionally $10^{\circ}$ away from the center to increase the visual display range contralateral to the hemisphere under study. All procedures were approved by the Harvard Medical Area Standing Committee on Animals.

\section{Visual stimuli}

Stimuli were presented on a ViewSonic P225f monitor (refresh rate, 100 $\mathrm{Hz}$ ) placed at a distance of $39 \mathrm{~cm}$, covering $55^{\circ}$ of visual space. All stimuli were generated using the Matlab toolbox Cogent Graphics, developed by John Romaya at the Wellcome Department of Imaging Neuroscience (http://www.vislab.ucl.ac.uk/cogent.php).

For the V2/V3 inactivation experiments, stimuli were circular patches of random dots (dot width $0.3^{\circ}$, dot density $1 \mathrm{dot} / \mathrm{deg}^{2}$ ) moving within a stationary window, placed at the center of each receptive field (RF). Dots first appeared stationary, then, after a $240 \mathrm{~ms}$ delay, moved coherently in the neuron's preferred direction (100\% coherence, infinite lifetime). Each stimulus was presented an average of seven times. For speed tuning experiments, the stimuli moved in the neuron's preferred direction at one of seven speeds $\left(1,2,4,8,16,32\right.$ or $\left.64^{\circ} / \mathrm{s}\right)$, and the diameter of each window was matched to the size of the RF center. For size tuning experiments, the dots moved at the neuron's preferred direction and speed as we varied the diameter of the stimulus window (size of $1,6,11,16,21,26$, and $\left.31^{\circ}\right)$.

Visual stimuli for the contrast experiments have been described previously (Pack et al., 2005). Briefly, stimuli were random dot fields (dot luminance of $139.5 \mathrm{~cd} / \mathrm{m}^{2}, \sim 0.1^{\circ}$ wide, density $0.5 \mathrm{dot} / \mathrm{deg}^{2}$ ) presented on a dim background $\left(2.2 \mathrm{~cd} / \mathrm{m}^{2}\right)$; equivalent contrast values were determined by the SD of the luminance (Moulden et al., 1990), equal to $9.8 \%$ for the high-contrast condition and $0.7 \%$ for low contrast.

For recordings made from areas V2 and V3 to confirm efficacy of the cooling method, visual stimuli consisted of single white bars swept across the receptive field $\left(1.5^{\circ}\right.$ wide on average $)$ at each site's preferred orientation.

\section{Extracellular recordings}

Our methods for extracellular recordings have been described previously (Pack et al., 2005; Ponce et al., 2008). Briefly, spike data were obtained from six monkeys ( $\mathrm{K}$ and $\mathrm{M}$ for cooling experiments; $\mathrm{G}$ for low-contrast speed tuning experiments and $\mathrm{C}, \mathrm{L}$, and $\mathrm{O}$ for the low-contrast size tuning experiments). Each monkey's MT region was located using structural magnetic resonance imaging and neuronal response properties, including the well known relationship between RF size and eccentricity (Van Essen et al., 1981; Desimone and Ungerleider, 1986) and high proportion of direction-tuned neurons. Within a given experiment, signals were first isolated using standard amplification and filtering (BAK Electronics) and receptive fields mapped using a small moving bar. Spike waveforms were digitized at $25 \mathrm{kHz}$ and stored to the computer's hard drive using a Cambridge Electronic Design 1401 system, and were subsequently analyzed with Spike2 software to confirm single-unit isolation and account for changes in the composition of multiunit signals. For the V2/V3 cooling experiments, the sampled MT receptive fields were centered within $2-34^{\circ}$ of the fovea $\left(14 \pm 7^{\circ}\right.$, mean \pm SD) and had an average width of $12.6 \pm 6^{\circ}$.

\section{Cooling devices and data collection}

The cooling loops (cryoloops) were made of 23-gauge stainless steel tubing with an attached thermistor, as detailed previously (Lomber et al., 1999). We placed three such loops adjacent to one another within the lunate sulcus covering an area of $\sim 8 \mathrm{~mm}$ deep (i.e., extending down into the sulcus) by $18 \mathrm{~mm}$ wide (i.e., from medial to lateral along the sulcus) (see Ponce et al., 2008, their Fig. 1). Experiments began with the collection of control data at physiological brain temperatures $\left(35-37^{\circ} \mathrm{C}\right.$ as measured by the cryoloops). After this block of control data, we initiated cortical cooling by pumping chilled methanol through the cryoloops, adjusting the flow rate until the devices reached temperatures between 2 and $6^{\circ} \mathrm{C}$ [corresponding to cortical temperatures of $<20^{\circ} \mathrm{C}$ within a distance of $1.5 \mathrm{~mm}$ from the loop (Lomber et al., 1999)]. To confirm that activity in the lunate sulcus was silenced during cooling, we performed a series of multiunit and single-unit recordings in the deep layers of the anterior bank of the lunate sulcus and observed a complete elimination of visually evoked activity, fully reversible within 1-2 min of flow cessation (Ponce et al., 2008, their Fig. 1d,e). Due to time limitations, we were not able to exhaustively map the efficacy of cooling over the entire expanse of cortex covered by our cryoloops. However, we were able to measure the extent of the V2/V3 "scotoma" by plotting the change in activity of each MT unit (represented by the blocking index, defined as $B I=1-\frac{R_{\text {max,inactivated }}}{R_{\text {max,control }}}$, where $R_{\max }=$ firing frequency at the preferred stimulus) as a function of its receptive field position. The region of the visual field showing the largest decrease in evoked activity was located within the central $10-15^{\circ}$ of the lower hemifield (contralateral to the inactivated lunate sulcus; see Ponce et al., 2008, their Fig. $2 c$ ), as predicted by the visuotopic representation of V2/V3 in the lunate sulcus (Gattass et al., 1981, 1988).

\section{Data analyses}

Spiking activity during the first $500 \mathrm{~ms}$ after motion onset was used to construct time-averaged speed and size tuning curves. ANOVAs were used to determine whether a neuron's activity was reliably modulated by variations in stimulus features. Visual discrimination indices were computed for each

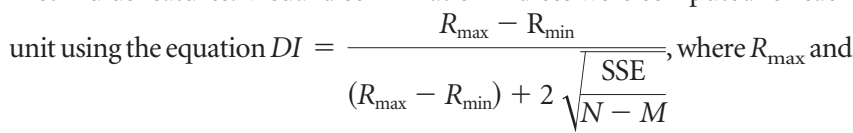
$R_{\min }$ are the responses to the preferred and null stimuli, SSE is the squared sum error around the mean response to each stimulus type, $M$ the number of trial types, and $N$ the number of trials.

Cooling experiments. For speed tuning tests, 57 of 61 single units and multiunits passed the above ANOVA criterion (ANOVA tests were performed on the square-root of the firing rates to homogenize the variance across different stimulus responses). Tuning curves with statistically significant modulation were fit (using the lsqcurvefit.m function in Matlab) with skewed Gaussian functions of the form, $R(s)=R_{0}+A \times e^{-\frac{\log [q(s)]^{2}}{2 \sigma^{2}}}$, where $q(s)=\frac{S+S_{\mathrm{o}}}{S_{\mathrm{p}}+S_{0}}, s=$ stimulus speed, $R_{0}=$ offset, $A=$ amplitude, $\sigma=$ Gaussian width, $s_{\mathrm{p}}=$ preferred speed, and $s_{0}$ is an offset value to prevent the logarithm from becoming unbounded. Fits were considered to be appropriate if they passed the $\chi^{2}$ goodness-of-fit test $(p>0.05)$, which determines whether the residual variance around the mean responses is significantly different from the residual variance around the fit. Eighty-eight percent (50 of 57) of all units passed this criterion before cooling; of these, 47 of 50 passed the criterion during cooling $(p>0.05)$. All parametric analyses are based on these 47 units ( 23 single units, 24 multiunits).

For size-tuning analyses, raw tuning curves were separately fit with two different functions: an error function (ErF), which is the integral of a Gaussian, of the form $R(s)=R_{0}+A_{e} \times \operatorname{erf}\left(\frac{s}{\alpha}\right)$, and a difference of error (DoE) function, which is the integral version of the "difference of Gaussians" model of center-surround interactions: $R(s)=R_{0}+A_{e} \times \operatorname{erf}\left(\frac{s}{\alpha}\right)-A_{i} \times \operatorname{erf}\left(\frac{s}{\alpha+\beta}\right)$. Units were classified as significantly surround-suppressed if the DoE function proved to be a statistically better fit than the ErF as determined by a sequential $F$ test, where significance was defined as $p<0.05$ (DeAngelis and Uka, 2003). In these cases, the preferred size was defined as the maximum value of the function; if units were well described by an ErF, the preferred size was taken as $1.163 \alpha$ (or $90 \%$ ) of the maximum function value. Surround suppression indices were 
defined as $S I=100 \times\left(\frac{R_{\mathrm{opt}}-R_{\text {largest }}}{R_{\mathrm{opt}}-S}\right)$, where $S=$ spontaneous activity, $R_{\text {opt }}=$ maximum response, and $R_{\text {largest }}=$ response to the largest stimulus size $\left(31^{\circ}\right)$.

Bootstrap analyses. To determine whether each unit's shift in preferred speed during cooling was significant, we performed a series of bootstrap analyses as follows. In the first and simplest variation, we resampled each unit's control (i.e., before cooling) spike counts (with replacement) and refitted a log-Gaussian to the data to obtain the new sample's preferred speed. We repeated this process hundreds of times $(n=500)$ and asked whether the observed cooling preferred speed fell outside the $95 \%$ range of the bootstrap distribution. In the second bootstrap variation, we attempted to replicate the effects of cooling in the speed tuning data using a two-stage bootstrap analysis: in the first stage, we computed a distribution of 500 gain and offset change values from every control/cooling pair of speed tuning curves (where gain is the ratio of tuning curve amplitudes derived from Gaussian fits Gain $=\frac{\text { Amplitude }_{\text {cooling }}}{\text { Amplitude }_{\text {precooling }}}$, and offset change was defined as offset $\Delta=$ Offset $_{\text {cooling }}-$ Offset $_{\text {precooling }}$ ). In the second stage, we resampled the control speed tuning data and applied a randomly sampled gain and offset value from the previous distribution to each spike count. This resulted in 500 new "cooling" speed tuning curves that could be compared with the measured cooling speed tuning curve. Measured cooling values were considered statistically significant if they fell outside of the $95 \%$ range predicted by the bootstrap. The final bootstrap analysis was a cross-modality variation of the preceding analysis, where the distributions of gain and offset values were derived using each neuron's direction tuning data and applied to the precooling speed tuning data.

Temporal dynamics. We computed the instantaneous preferred value (speed or size) and tuning curve amplitude for each unit as follows. We calculated the average number of spikes per millisecond for each condition (stimulus speed or size) and convolved this spike train with a Gaussian function (4 ms width) starting at motion onset. Response latency was defined as the time point after motion onset when the response magnitude reached 2 SDs above the baseline activity. Instantaneous tuning curve amplitude was defined as the difference in average spike count (for each ms bin) measured at the unit's time-averaged preferred and null stimulus values. The instantaneous preferred value was defined as the stimulus value that elicited the highest number of spikes at each bin. For smoothing, each unit's instantaneous temporal vector was fit with a least-squares spline (Matlab function spap2.m, knots $=3$, order $=4$ ) starting $50 \mathrm{~ms}$ after stimulus motion onset.

Pursuit experiments. Each trial began with the onset of two stationary targets; a centrally placed fixation target as described above and a second circle $\left(1^{\circ}\right.$ diameter) placed at $\left[-3,-3^{\circ}\right]$ or $\left[+3,-3^{\circ}\right]$. The animals were required to maintain fixation within $2^{\circ}$ of the central point. After an initial fixation period between 1500 and $2000 \mathrm{~ms}$, the central fixation target disappeared and the eccentric target began to move toward or away from the fovea. The monkeys were rewarded if they maintained fixation on the second target during the duration of the ramp $\left(15^{\circ} \mathrm{long}\right)$. This task frequently required them to make saccades to the target; we allowed for a $400 \mathrm{~ms}$ grace period between fixation point disappearance and motion onset.

Eye position and velocity (analog differentiator: low-pass, $-3 \mathrm{~dB}$ at 50 $\mathrm{Hz}$ ) were digitized and stored to disk at $250 \mathrm{~Hz}$ for off-line analysis. We only analyzed trials that had been successfully completed (15-26 trials per condition). Trials were manually discarded if any saccades or blinks occurred within the first 100 milliseconds after ramp onset. Saccades occurring after this period were detected and replaced with Not-a-Number values (NaNs), which were treated as missing values during subsequent analyses. Pursuit onset was found using a modification (Madelain and Krauzlis, 2003) of the algorithm published by Carl and Gellman (1987): briefly, onset was defined as the intersection between two regression lines fit to the individual eye velocity traces. The first line was fit to the baseline (first $50 \mathrm{~ms}$ after motion onset) and the second line was fit to the response eye velocity data (first $30 \mathrm{~ms}$ after the point where eye velocity exceeded 4 SDs above
A

B

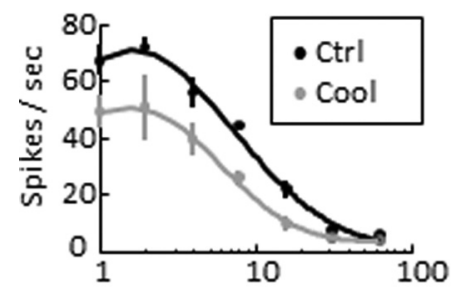

C

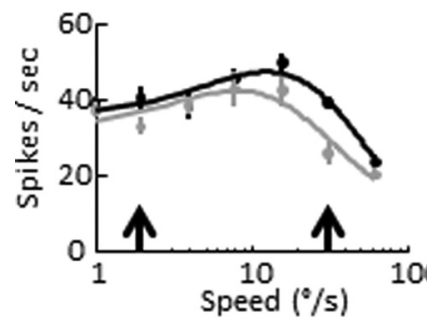

D
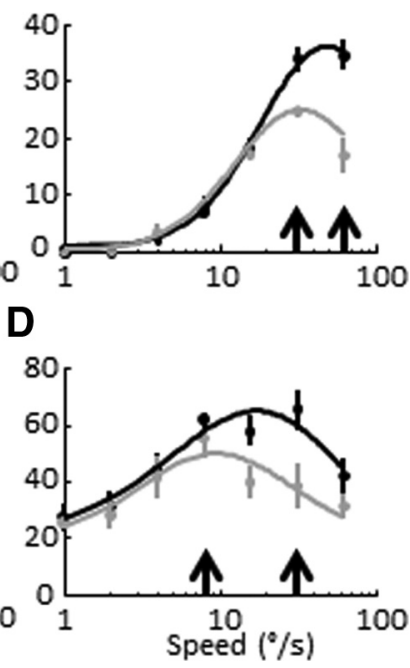

Figure 1. $A-D$, Speed tuning curves computed from the responses of four different MT neurons (three single units, one multiunit). Each neuron is represented by a pair of tuning curves showing its mean control and cooling responses ( \pm SEM). Some cells responded best to slow speeds $\left(\boldsymbol{A}\right.$, preferred speed $\left.1.6^{\circ} / \mathrm{s}\right)$, others to faster speeds $(\boldsymbol{B}, 50.2 \%)$, and others to medium speeds $\left(\boldsymbol{C}, \boldsymbol{D}, 12.8^{\circ} / \mathrm{s}, 17.5^{\circ} \mathrm{s}\right)$. The median control speed across the population was $15.2^{\circ} \mathrm{s}$. Curves show the log Gaussian fits, points and vertical lines show the mean and SEM, and black and gray colors represent precooling and cooling responses. Black arrows indicate equivalent responses before cooling (see Speed tuning in main text).

average baseline activity). The slope of this second regression line was used as a measure of acceleration. Statistical significance was determined with a two-way ANOVA using temperature and stimulus speed as factors; the square roots of eye acceleration values were used to normalize variance.

\section{Results}

\section{Speed tuning}

MT neurons generally demonstrated decreased firing rates during V2/V3 cooling (Figs. $1 A, 2 A$ ). We quantified this with the blocking index (BI) (see Materials and Methods): positive BI values indicate a decrease of firing rate during cooling, while negative BI values indicate an increase. Using the amplitude values from the skewed Gaussian fits to speed tuning data, we found that the median speed blocking index was $0.16 \pm 0.06$ (SE via bootstrap, 1000 iterations). For individual recording sites this reduction in activity could translate to both changes in log Gaussian function offset and/or tuning curve amplitude (Fig. 1). The median offset change was zero ( $p=0.57$, Wilcoxon signed rank rest, $n=57$; mean was 1.3 spikes/s; if we excluded neurons with no measurable offset activity before cooling, the median change was -4 spikes/s; $p=0.18$, signed rank test), while the median amplitude change (cooling minus control) was -7 spikes/s ( $p=0.013$, signed rank test, mean -10 spikes/s; Fig. 2 A).

The overall reduction in speed tuning gain indicates a decrease in the extent to which varying stimulus speed modulates the firing rates of MT neurons when the indirect pathway is inactivated. Whether this reduced modulation results in a reduction in the ability of these neurons to faithfully encode speed information depends on the extent to which response variability is affected. We thus used a measure of tuning curve amplitude that is normalized by response variability, the discrimination index (DI) (see Materials and Methods). We previously showed that the median discrimination index change for direction tuning was 
A
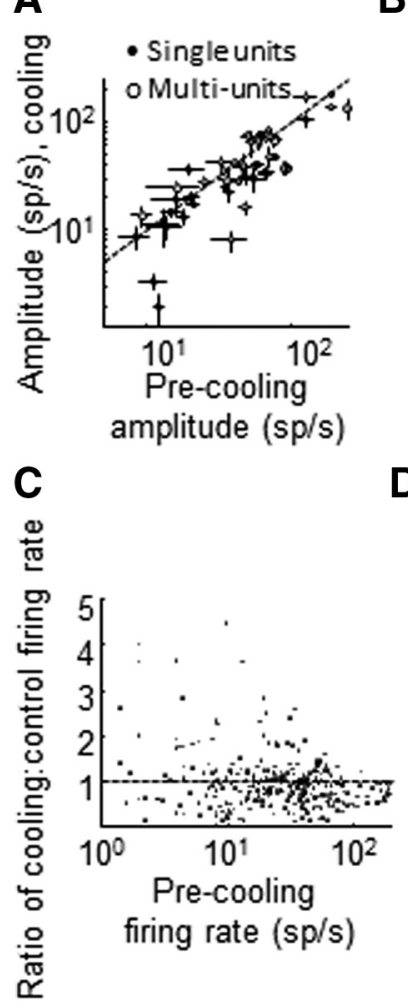

B

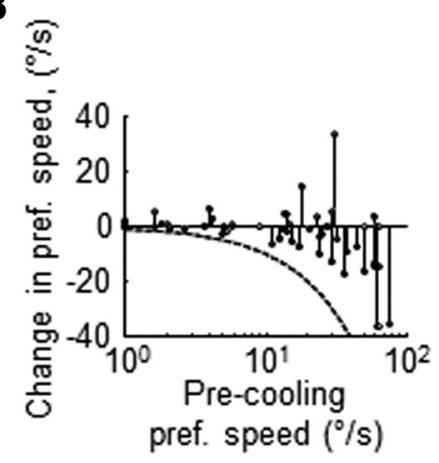

D

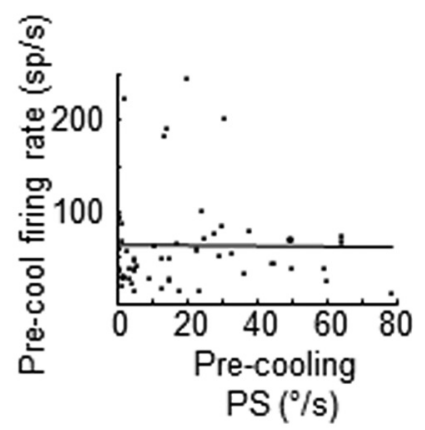

Figure 2. MT speed tuning during V2/V3 inactivation. $\boldsymbol{A}$, Speed tuning curve amplitude values measured before and during cooling ( \pm SEM). Closed dots, single units; open dots: multiunits. $\boldsymbol{B}$, Change in preferred speed during cooling as a function of control preferred speed. Closed dots, single units; open dots, multiunits. Broken line shows the theoretical maximal decrease in preferred speed. $C$, Change in firing rate as a function of control firing rate. Each dot shows the ratio (cooling/control) of average spike counts to a given trial type for all neurons. $\boldsymbol{D}$, Control firing rate measured at each unit's preferred speed. Black line shows the correlation line.

only -0.04 (cooling minus control DI), whereas the median loss in discriminability for disparity tuning was nearly four times larger $(-0.15)$. The median change in speed DI was -0.07 , intermediate between direction and disparity, but closer to the former. Thus we find that speed tuning is less affected by inactivation of the indirect pathways than is tuning for binocular disparity.

Visual inspection revealed that many neurons undergoing a gain change also demonstrated unequal cooling effects in response to different stimulus speeds. In particular, responses to faster speeds were often disproportionately suppressed by inactivation of V2/V3. For example, some neurons that responded with comparable firing frequencies to two different speeds under control conditions showed a steeper response reduction to the faster speed during cooling (Fig. $1 B$, black arrows: see responses to $32 \%$ vs $64 \%$ sefore and during cooling; Fig. $1 C$, note responses to $2 \%$ s vs $32 \%$, or Fig. $1 \mathrm{D}$, responses to $8 \%$ s and $32 \%$ s). This had the effect of shifting the preferred speed of these units toward lower values; for two of the units above, the preferred speeds shifted from $50 \%$ s to $34 \%$ and from $13 \%$ s to $8 \%$ s. Overall, $66 \%$ of all units (31 of 47) showed a decrease in preferred speed (30\%, 14 of 47 , were statistically significant changes, see Materials and Methods for the first bootstrap analysis). A stem plot showing the relationship between each unit's control preferred speed and its corresponding change during cooling indicates an overall shift toward slower preferred speeds (Fig. $2 B$ ).

The most parsimonious interpretation of this finding is that MT becomes less responsive to larger spatial displacements in the

absence of V2 and V3 inputs, but there are other possible explanations for this effect. First, it is conceivable that higher firing rates are associated with proportionately greater firing rate reductions during cooling and the neurons with higher firing rates also prefer higher speeds. While there is indeed an inverse relationship between control firing rate and the change in firing rate during cooling (Fig. 2C) there was no relationship between control preferred speed and control firing rates $\left(r^{2}=0.0046, p=\right.$ 0.6519; Fig. 2D). In other words, before cooling, neurons with higher preferred speeds did not fire more vigorously than neurons with lower preferred speeds. This conclusion was confirmed with a partial correlation test (Zar, 1999) that quantified the relationship between control preferred speed and the change in preferred speed while controlling for firing rate $(r=0.27)$.

Another possible explanation for this decrease in the population preferred speed is a simple ceiling effect: any one unit may experience a random change in preferred speed during cooling, toward either higher or lower speed values; as a consequence, units responding best to our highest tested speeds $(32-64 \%$ s) would then show a net decrease in preferred speed because we did not probe for responses to higher values $\left(>64^{\circ} / \mathrm{s}\right)$. Given the significant proportion of MT cells that respond to higher speeds than we tested (Lagae et al., 1993), this is a real possibility that we sought to exclude in two ways. First, if neurons were undergoing random changes in preferred speed, we should also have observed a floor effect at the lower end of the preferred speed distribution, where the result would be an increase in preferred speed. In this event, the stem plot in Figure $2 B$ would demonstrate a negative linear relationship between control preferred speed and its corresponding shift in preferred speed, characterized by leftward increases in preferred speed. In fact, the median preferred speed change for the smallest four speeds tested was $0.0 \pm 0.3^{\circ} / \mathrm{s}$ (SEM via bootstrap). Second, we confined our analysis to units with middle-range preferred speed values (defined as $>4 \%$ and $\leq 20 \%$ $\mathrm{s})$, where the random hypothesis would predict a normal distribution of preferred speed changes around zero. In this subset of the data, we found that only five of 15 units exhibited an increase in preferred speed, while the rest showed a decrease. These results are not consistent with a floor or ceiling effect.

We also considered whether changes in eye movements during cooling could explain this trend. Although we discarded any trials during which the monkeys broke fixation, it was important to make sure that there were no small gaze displacements within the fixation window, either during the fast or slow speed trials, which could account for the above effects. We found no significant differences in average eye speed or microsaccade frequency as a function of stimulus speed before or during cooling (eye velocity: $p=0.73$, two-way ANOVA; microsaccade frequency: $p=0.24$, two-way ANOVA; speed and temperature used as factors).

We also used a series of bootstrap analyses to assess whether the speed tuning shift are the result of noisy reductions in firing rate (i.e., simple gain and offset changes). First, we resampled the control and cooling speed tuning data to compute a distribution of gain and offset change values that describe the changes observed in each neuron's speed tuning curves. In a subsequent bootstrap, we resampled the control speed tuning again and applied a gain and offset (sampled randomly from the distributions obtained with the preceding bootstrap) to each spike count. This new dataset was then fit with a log Gaussian function to obtain a preferred speed, and the actual preferred speed measured during V2/V3 inactivation was compared with the resulting distribution. Despite these simulated noisy reductions in firing rate, $30 \%$ of 
units continued to demonstrate statistically significant changes in preferred speed. In a related analysis, we first combined the control and cooling datasets of each neuron and resampled the combined data, with replacement, to generate two new tuning curves (a "control" and "cooling" curve); we then took the difference in preferred speed between each pair of curves and generated a distribution of differences to compare with the actual, measured difference in preferred speed. We found that $28 \%$ of the units showed reductions in speed below the fifth percentile of these simulated distributions. In our previous paper exploring V2/V3 cooling, we also used a third type of bootstrap analysis, which manipulated control data using gain and offset changes measured in response to a different modality (direction; see supplemental Fig. S1, available at www.jneurosci.org as supplemental material). We replicated this analysis here and confirmed that direction tuning changes could not explain the above effect. Thus we conclude that many of the changes in preferred speed are a consequence of mechanisms other than a simple firing rate reduction.

\section{Pursuit eye movements}

There is strong evidence that MT signals are used to guide the initiation of smooth pursuit eye movements (Newsome et al., 1985; Groh et al., 1997), so we predicted that the reduction in preferred speed across the MT population observed during V2/V3 inactivation would have consequences for pursuit eye movements. We thus trained two monkeys to perform a simple spot pursuit task (monkeys $\mathrm{M}$ and J). Each monkey was required to fixate a central spot while a second stationary target was presented either within the V2/V3 "scotoma" (in the lower visual quadrant contralateral to the cooled lunate sulcus; coordinates $[-3,-3]^{\circ}$ ) or outside of it (within the lower visual quadrant ipsilateral to the sulcus; coordinates $\left.[+3,-3]^{\circ}\right)$. After a random period of fixation, the central fixation target disappeared and the eccentric target began to move toward or away from the fovea at one of five different speeds: $10,20,30,40$ or $50 \%$ s. The monkey was required to track this target within a $7^{\circ}$ window for the duration of the ramp. As shown in previous studies (Lisberger and Westbrook, 1985), monkeys showed increasing presaccadic eye velocity as a function of stimulus speed (Fig. 3A) for targets moving toward the fovea. Visual inspection of these traces revealed a slight decrease in eye velocity during cooling, especially at higher stimulus speeds. To quantify these effects, we fit a regression line to individual-trial eye velocity traces to determine each trial's ocular acceleration (Fig. 3B), and these were averaged and plotted as a function of stimulus speed (Fig. $3 C$ ). We found that the open-loop eye acceleration of both monkeys was reduced during V2/V3 inactivation, especially for the highest stimulus speed (monkey J: 30\% reduction; monkey M: 46\%); two-way analyses of variance using temperature and stimulus speed as factors (see Materials and Methods) showed that the effect of temperature was significant in one monkey and approached significance in the other ( $p$ values $=0.01$ for monkey $\mathrm{M}$ and 0.08 for monkey $\mathrm{J})$.

We obtained rewarming data from one monkey $(\mathrm{M})$ and confirmed that these effects were reversible (Fig. 3C, broken line). As commonly seen in eye movement experiments, there were some idiosyncratic differences between both monkeys: monkey $\mathrm{M}$ showed the largest decrease in eye acceleration in response to $50 \%$ trials, while monkey J showed larger decreases to $30-40 \%$ trials. However, both monkeys failed to exhibit any significant pursuit changes to the slowest speed. This behavioral effect is thus consistent with the neurophysiological findings and another il-

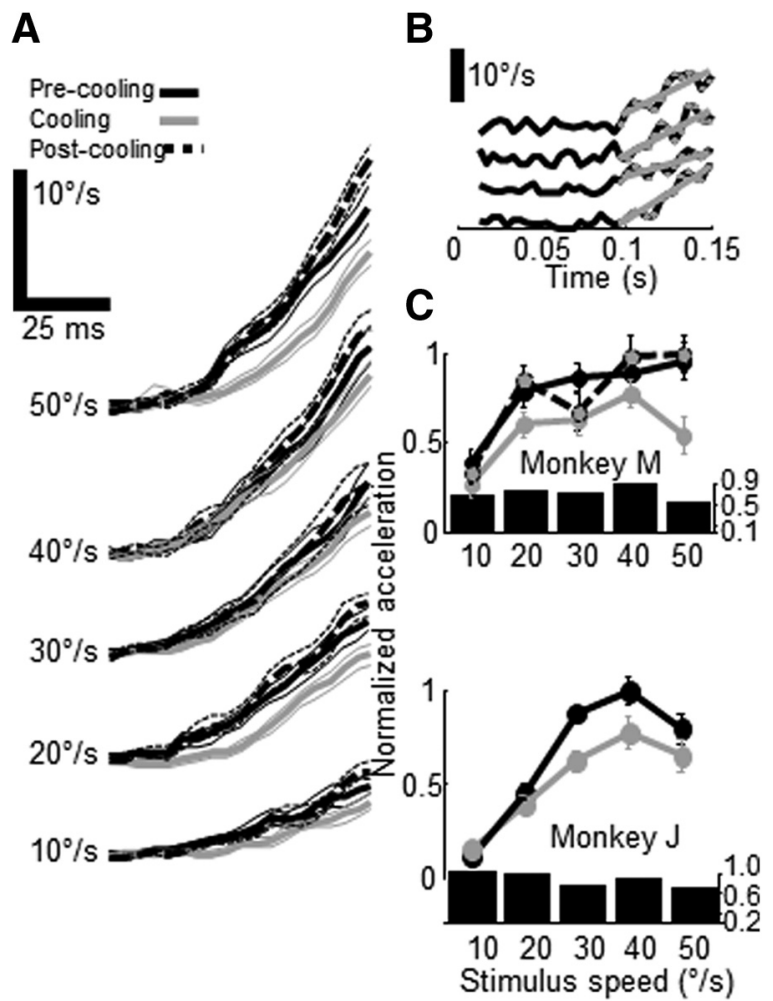

Figure 3. Effects of V2/V3 cooling on smooth pursuit. $A$, Average eye speed ( \pm SEM) in response to pursuit targets moving at different speeds $\left(10,20,30,40\right.$ and $\left.50^{\circ} / \mathrm{s}\right)$ before, during and after cooling (black, gray and broken black lines). Average onset of presaccadic pursuit for all trials was $92 \mathrm{~ms}$. $\boldsymbol{B}$, Individual eye speed trials (black) fit with linear regression lines (gray) used to compute acceleration. Data points used for regression highlighted in gray. $\boldsymbol{C}$, Normalized acceleration ( \pm SEM) as a function of target speed for two monkeys $(M, J)$ measured before, during and after cooling (black, gray and black-gray circles). Bar plots indicate the ratio of mean cooling/control eye acceleration as a function of stimulus speed.

lustration of the close relationship between MT population activity and the initiation of smooth eye movements.

\section{Size tuning}

Forty-five MT units were tested for size tuning properties before and during V2/V3 inactivation; of these, 42 were significantly modulated by stimulus size ( $p<0.05$, one-way ANOVA) before cooling and 40 during cooling; 35 units were well fit with both ErFs and DoE functions $\left(p>0.05, \chi^{2}\right.$ test). The size-tuning curves were analyzed for changes in three features: gain, preferred size and surround suppression (SS) (see Materials and Methods). As expected from the preceding speed tuning data, the overall level of activity for the population decreased during V2/V3 cooling (27 of these neurons were also part of the speed tuning analysis above). The median size-tuning blocking index for the 35 units was $0.17 \pm 0.08$ (SE via bootstrap, 1000 iterations) with a corresponding decrease in median tuning curve amplitude of 7 spikes/s ( $p<0.01$, signed rank test; Fig. $4 B, C$ ). While we noted some interesting shape changes in individual single-unit and multiunit tuning curves during cooling (Fig. 4), leading to changes in the preferred size and SS, the preferred size did not change significantly across the population (control median preferred size from the parametric fits was $9.8^{\circ}$, with a median increase during cooling of $2.4 \pm 1.8^{\circ} ; p=0.11$, signed rank test). We found 11 units that were significantly surround-suppressed as assessed by the sequential $F$ test $(p<0.05)$. The average SS for these cells was $42 \%$; during cooling, five units lost statistically 
A
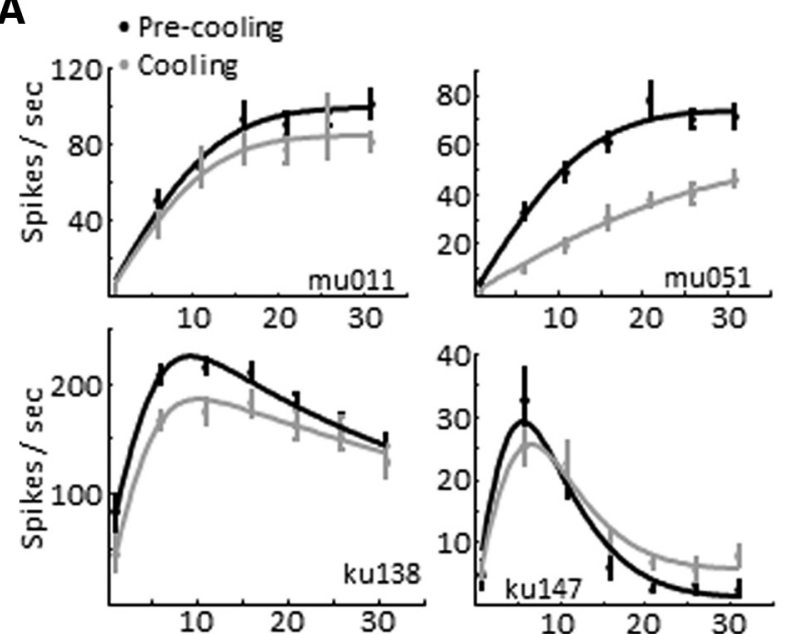

Stimulus diameter $\left({ }^{\circ}\right)$

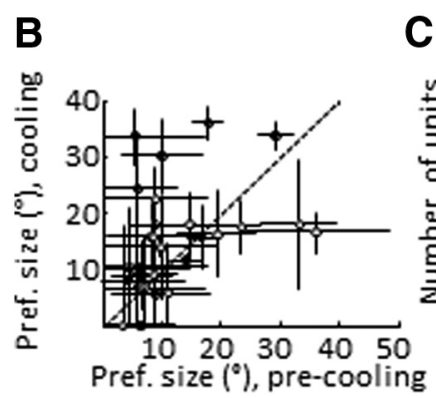

C

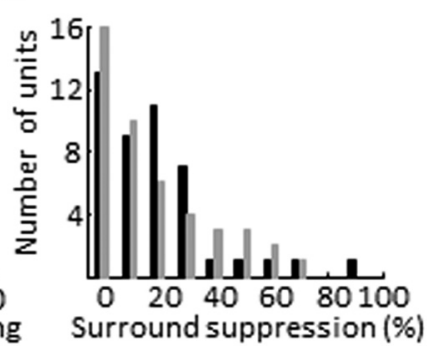

Figure 4. MT size tuning during V2/V3 inactivation. $A$, Size tuning curves computed from the responses of four different MT neurons. Each neuron is represented by a pair of tuning curves showing its control (black) and cooling (gray) responses. Cells differed in the functional responses of the modulatory surround: some being facilitatory (top row) while others exhibited suppressive properties (bottom row). Vertical lines show the mean and SEM. Curves are either error function or difference of error function fits. $\boldsymbol{B}$, Preferred size measured before and during cooling ( \pm SEM) (via bootstrap, 500 iterations). Open symbols, multiunits, closed symbols single units. C, Distribution of surround suppression values before and during cooling.

significant SS and five of the remaining six units showed a decrease in SS (average decrease of $9 \%$ ), with an associated median increase in cooling preferred size of $2^{\circ}(p=0.31)$. When we included all neurons in our sample, regardless of statistical significance per the sequential $F$ test $(n=42)$, we found a nonsignificant increase in surround suppression of $1.4 \%(p=0.26$, Wilcoxon signed rank test). The units that maintained strong surround inhibition during cooling also demonstrated a $7 \%$ net decrease in the inhibitory Gaussian. Only one unit became significantly more surround suppressed during cooling. Thus the majority of our surround-suppressed cells demonstrated a reduction in the strength of the suppressive surround, but the effect was rather weak.

\section{Comparison to low-contrast effects}

Thus far we have demonstrated that reversibly inactivating the $\mathrm{V} 2 / \mathrm{V} 3$ inputs to MT produces a decrease in the preferred speed as well as a modest reduction in surround suppression. Similar changes have been reported previously in response to visual stimuli of low contrast (Pack et al., 2005; Huang et al., 2008), so we were curious to know whether there were other similarities. Of course, lowering stimulus contrast has numerous effects on earlier stages of visual processing, including complex effects on retinal responses (Shapley and Victor, 1978), changes in V1 receptive fields, including altered surround suppression (Levitt et al., 1994a; Polat et al., 1998; Kapadia et al., 1999; Sceniak et al., 1999; Cavanaugh et al., 2002a,b), and changes in temporal filtering (Albrecht, 1995; Frazor et al., 2004). We are obviously unable to address the contribution of these factors at present. One thing that the two treatments (i.e., cooling and lowering contrast) do have in common is that they both reduce the overall firing rate of MT neurons, and it is conceivable that this alone is sufficient to produce the observed changes in MT.

Ideally, one would like to compare the effects of contrast and cooling on the same MT neurons; however, we were unable to record for sufficiently long periods of time from single neurons to perform both comparisons. We thus explored this issue by comparing our cooling data with results from separate contrastmanipulation experiments. Our main goal was to examine the similarities between the effects of V2/V3 cooling and low visual contrast on MT neurons and to subsequently identify underlying differences in both datasets, if any.

We first confirmed some similarities between the two datasets: for example, both groups of neurons showed the same pattern of preferred speed reduction as a function of control preferred speed, a pattern that held even after controlling for each neuron's firing rate (supplemental Fig. S2 A, available at www.jneurosci. org as supplemental material). Overall, these changes were greater for the speed contrast data (even when controlling for mean firing rate) but Krekelberg et al. (2006) showed that intermediate contrast levels lead to intermediate preferred speed reductions, raising the possibility that V2/V3 cooling is functionally equivalent to a smaller reduction in contrast than the one used in our previous contrast experiments.

However, the two datasets also differed in interesting ways. First, while low contrast reduced the overall population mean firing rate, it also increased firing rate responses to slower speeds for many neurons (Pack et al., 2005; Krekelberg et al., 2006), and this effect was never seen in our cooling data. As a result, during low contrast, there was no net decrease in population firing rate at lower stimulus speeds, and this effect is true for intermediate contrasts as well (see also Krekelberg et al., 2006). By comparison, $\mathrm{V} 2 / \mathrm{V} 3$ cooling decreased mean firing rate for all stimulus speeds, without the slow-speed facilitation (supplemental Fig. S2 B, $C$, available at www.jneurosci.org as supplemental material). Second, there were interesting differences in the temporal dynamics of MT neurons between the two conditions. As expected from previous studies (Raiguel et al., 1999) we found that low contrast delayed both the response latency [high contrast, $88 \pm 4 \mathrm{~ms}$ (SEM); low contrast, $140 \pm 11 \mathrm{~ms}$; supplemental Fig. S2 D, available at www.jneurosci.org as supplemental material] and the latency of each unit's maximum tuning curve amplitude (high contrast, $153 \pm 26$, low contrast, $311 \pm 26$; supplemental Fig. $\mathrm{S} 2 E$, available at www.jneurosci.org as supplemental material), whereas indirect pathway inactivation did not cause a significant delay in either response (control, $79 \pm 5 \mathrm{~ms}$; cooling, $80 \pm 7 \mathrm{~ms}$; supplemental Fig. S2 D, available at www.jneurosci.org as supplemental material) or maximum amplitude latency (control, $213 \pm$ 23; cooling, $193 \pm 20 \mathrm{~ms}$; supplemental Fig. S2 E, available at www.jneurosci.org as supplemental material).

We also found a difference in the time course of speed tuning. As noted in previous reports, during normal conditions MT neurons initially produce a large transient response that is tuned to higher speeds, followed by a smaller sustained response tuned to slightly lower speeds (Lisberger and Movshon, 1999) (Fig. 5C,D). We found that this transient disappeared for low-contrast conditions but not for V2/V3 inactivation (Fig. $5 A, B$ ). As a result, the mean difference in speed preference between high- and low- 
A
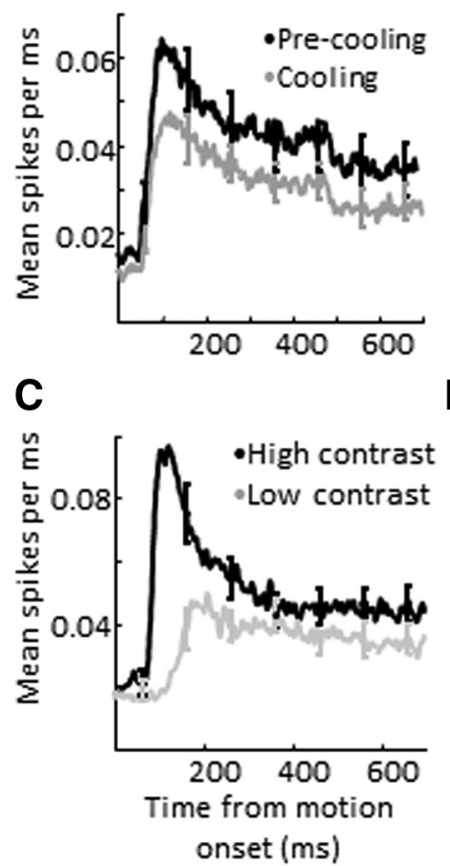

B

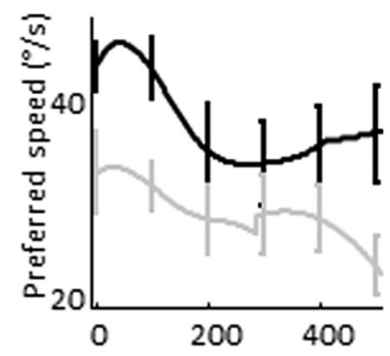

D

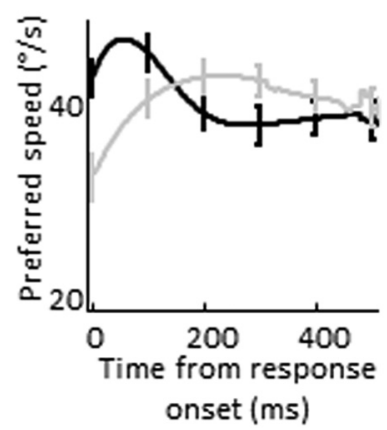

Figure 5. Temporal dynamics of speed tuning in MT. $\boldsymbol{A}$, Average response ( \pm SEM) before (black) and during cooling (gray). $\boldsymbol{B}$, Average preferred speed ( \pm SEM) for high-pass units (defined as units with time-averaged preferred speed above $32 \%$ ), aligned by each neuron's response onset, before (black) and during (gray) cooling. C, Average response ( \pm SEM) for high (black)- and low (gray)-contrast conditions. D, Average preferred speed ( \pm SEM) for high-pass units (defined as units with time-averaged preferred speeds above $32 \%$ ) during high (black)and low (gray)-contrast conditions, aligned by response onset. Broken line shows the mean preferred speed difference (pre-minus cooling).

contrast conditions peaked early after response onset and decreased over time. In comparison, the average difference in preferred speed between control and cooling conditions remained nearly constant throughout the entire trial. These changes in the transient response were quantified using a transient-sustained ratio (TSR) (defined as the ratio of the largest mean firing rate in the first $250 \mathrm{~ms}$ after motion onset, divided by the mean firing rate in the interval from $300 \mathrm{~ms}$ to the end of the trial). During the cooling experiments, the median TSR for all neurons increased by 7\% ( $p<0.01$, paired Wilcoxon rank sum test, $n=61$ ), while that for neurons in the contrast experiments decreased by $64 \%(p<0.001, n=40)$. While the underlying mechanisms accounting for these differences are unclear, they do help to disambiguate the contributions of the indirect pathways to speed processing in MT and further argue against a nonspecific, firing rate-dependent effect.

We also compared the temporal dynamics of size tuning between low-contrast and V2/V3 cooling conditions. For this analysis, we chose a subset of surround-suppressed neurons (any unit with surround suppression above $20 \%$ ) to explore the change in preferred size across low-contrast/cooling conditions (18 units from cooling experiments, 133 units from contrast experiments). As with the speed tuning data, we found an inverse relationship between contrast and response latency, whereas V2/V3 inactivation had no effect (Fig. 6A,C). We found that the temporal dynamics of preferred size were similarly flat for both datasets. Thus, for the case of size tuning, any cooling effects observed may be consistent with a relatively nonselective reduction in firing rate.

A

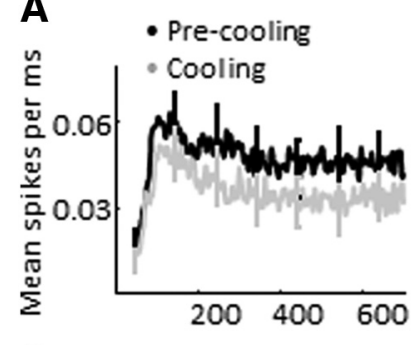

B
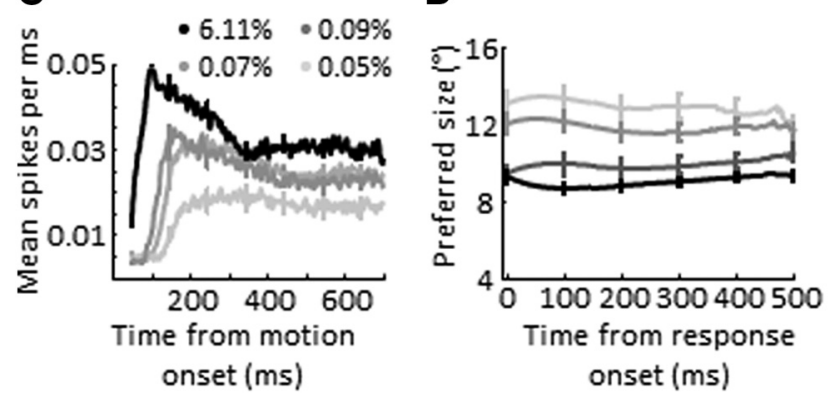

Figure 6. Temporal dynamics of size tuning in MT. $\boldsymbol{A}$, Average response ( \pm SEM) for all units with surround suppression $>20 \%$ before (black) and during cooling (gray). $\boldsymbol{B}$, Average preferred size ( \pm SEM) for all units with surround suppression $>20 \%$ ), aligned by each neuron's response onset, before (black) and during cooling (gray). C, Average response ( \pm SEM) for all units with surround suppression $>20 \%$ during high (black)- and low (gray)-contrast conditions (RMS contrast levels of $0.05,0.07,0.09$, and 6.11). $D$, Average preferred size ( \pm SEM) for all units with surround suppression $>20 \%$, aligned by response onset, during high (black)-and low (gray)-contrast conditions.

\section{Mixing of input streams at the single neuron level}

We also considered the possibility that the indirect inputs to MT were functionally segregated. MT is known to contain columnar organizations for both direction (Albright, 1984) and binocular disparity (DeAngelis and Newsome, 1999), as well as a noncolumnar clustering of preferred speed (Liu and Newsome, 2003). Insofar as the V2/V3 inputs to MT were nonrandom with respect to these levels of organization, one would predict differences in the efficacy of cooling as a function of the control tuning properties of the neuron. For example, given the finding of DeAngelis and Newsome (1999) that some regions within MT contain neurons with relatively poor binocular disparity tuning, and our previous finding that $\mathrm{V} 2 / \mathrm{V} 3$ inputs strengthen disparity tuning in MT neurons (Ponce et al., 2008), one might expect to find a decreased efficacy of cooling for neurons that had low discrimination indices for binocular disparity before cooling.

We analyzed our data in two different ways to test for possible inhomogeneity in inputs from the indirect pathway. First, we correlated control neuronal discrimination indices with the efficacy of cooling as measured in two different ways-either as the median or the maximal blocking index across all stimulus conditions. In all cases, we found no significant correlations with respect to tuning for direction, speed or binocular disparity. As a second measure, we divided our neuronal population into thirds based on the efficacy of cooling, as measured by the blocking index, and compared the top and bottom thirds with respect to discrimination indices, as well as the distributions of preferred values for speed, direction, size and binocular disparity. Again, we found no significant differences, suggesting that the combined inputs from V2 and V3 do not provide patchy inputs to MT that respect any of the known levels of organization. Since we simultaneously inactivated both input regions, it remains possible that 
the input from each region alone is segregated, but that the two together are complementary.

\section{Discussion}

Our previous study demonstrated that inactivation of the indirect pathways led to an overall decrease in MT activity and that this change was associated with a disproportionate degradation of binocular disparity tuning compared with that for direction (Ponce et al., 2008). Our current findings show that this manipulation also causes a population shift favoring slower speeds and a small decrease in surround suppression. On the whole, the changes we observed for binocular disparity tuning were the largest, with those in tuning for direction, speed and size considerably smaller. Thus, to a first approximation, our results suggest that the indirect pathways to MT are mainly specialized for conveying information about depth. At present we cannot rule out the possibility that they are also conveying more subtle, but equally important, information such as signals related to border ownership (Baumann et al., 1997; Zhou et al., 2000; Qiu and von der Heydt, 2005) that would allow MT neurons to preferentially represent the motion of intrinsic terminators (Duncan et al., 2000; Pack et al., 2004). As we have already treated the issue of indirect pathways and binocular disparity previously (Ponce et al., 2008), we will focus the remainder of the discussion on the present results concerning speed and size tuning.

The shift in speed tuning toward slower preferred speeds was not a result of neurons with faster preferred speeds (high-pass neurons) having larger control firing rates, as neurons with slow preferred speeds fired as robustly as high-pass cells. The shift could not be explained by a ceiling effect either, because the same trend was observed within the subset of neurons preferring intermediate speeds as well. Our results are superficially similar to previously reported effects of low-contrast stimuli on MT speedand size-tuning (Pack et al., 2005; Krekelberg et al., 2006); however, we also found significant differences in the dynamics of the different responses, particularly with respect to speed tuning. This leads us to conclude that the indirect pathways carry information that contributes significantly to MT neurons' ability to respond to faster speeds.

This interpretation raises several questions. First, it has been reported that the distributions of preferred speeds in striate cortex (V1) and MT are largely overlapping. Churchland et al. (2005) used random dot patterns to determine the maximum spatial step that can still result in V1 and MT directional responses, and found them to be similar between the two areas. Our laboratory used reverse correlation techniques to characterize the spatiotemporal receptive fields in V1 and MT, and found that the spatial scale of the interactions was quite similar between both visual areas (Pack et al., 2006). These results suggest that speed tuning in MT may be largely inherited from V1 neurons. In neither study, however, was it demonstrated that any particular V1 neuron actually provided direct input to MT. Given that a very select subset of V1 neurons projects to MT (Movshon and Newsome, 1996) and that direct-projecting neurons are intermingled with, but largely distinct from, those providing input to the indirect pathways (Sincich and Horton, 2003), it is also possible that the V1 neurons responding to larger spatial steps may preferentially project to MT by way of V2 and/or V3. Indeed, our results suggest that this may be the case. This may also explain why a larger proportion of MT neurons prefer faster speeds than do V1 cells (Mikami et al., 1986; Churchland et al., 2005; Priebe et al., 2006), and why V1 and MT spatiotemporal maps frequently fail to predict MT speed preferences, particularly for neurons tuned to faster speeds (Pack et al., 2006). On average, V2 and V3 neurons have coarser spatial frequency preferences (Foster et al., 1985), respond to faster speeds (Burkhalter and Van Essen, 1986) and have larger receptive fields (Orban et al., 1986) than those in V1, features that may confer an advantage in the integration of larger, spatially discrete displacements and thus responses to faster speeds, provided there are no systematic changes in tuning for temporal frequency. We and others have shown that the average maximal displacement for direction tuning in MT is slightly larger than that in V1 (Churchland et al., 2005; Pack et al., 2006). While these maximal displacement distributions are largely overlapping, it is plausible that the indirect pathways account for the difference.

Our treatment of speed tuning relied on the systematic variation of spatial information (position shifts of different magnitudes) while keeping the temporal interval constant (frame rate of $100 \mathrm{~Hz}$ ). Thus our interpretation of the role of the indirect pathways on speed tuning is restricted to the spatial properties of receptive fields, but what about their temporal features? Previous cat and monkey studies have characterized speed tuning using sine-wave gratings with varying temporal and spatial frequencies. In this framework, neurons whose preferred speeds vary as a function of spatial frequency are not technically tuned for speed (because they do not shift their preferred temporal frequency to maintain the same spatiotemporal ratio). These neurons are described as having separable tuning for spatial and temporal frequency: a Fourier plot showing response magnitude as a function of spatial and temporal frequencies would show independence of response. Most simple V1 neurons demonstrate separable tuning and thus are not strictly tuned for speed (Movshon, 1975; Tolhurst and Movshon, 1975; Foster et al., 1985; McLean and Palmer, 1994; Priebe et al., 2006); lateral geniculate cells show nonseparable spatiotemporal tuning, but it is nonspecific and does not represent speed (Hicks et al., 1983; Derrington and Lennie, 1984). In contrast, a small but significant proportion of V1 complex cells, V2 and MT neurons show a preference for a given ratio of temporal and spatial frequencies as expected for true speed tuning (Bisti et al., 1985; Foster et al., 1985; Levitt et al., 1994b; Priebe et al., 2003, 2006). Further, Priebe et al. (2003) showed that MT neurons become more distinctly speed-tuned as different spatial frequencies are added together-either by superimposing multiple sine-wave gratings or by using squarewave gratings or random dot patterns-while V1 neurons do not show this nonlinearity (Priebe et al., 2006). Our present work does not lead to strong predictions about the effects of V2/V3 inactivation on these spatiotemporal properties of MT neurons. However, given that these properties are well represented in MTprojecting V1 complex neurons (Movshon and Newsome, 1996; also see supplemental information in the study by Priebe et al., 2006), and that we failed to see any significant changes in speed tuning bandwidth, it is unlikely that MT neurons would become less speed-tuned (i.e., show less dependence of spatio-temporal responses, although some neurons might show offset changes toward lower preferred speeds). It is plausible that in the absence of V2/V3 input, the distribution of preferred spatial frequencies in MT would shift toward higher values (given a loss of coarsefrequency tuning). These are questions that merit further investigation.

Thus far we have considered only feedforward pathways. It is important to realize, however, that inactivation of all layers within V2 and V3, as we have done with the cooling technique, will also affect other pathways, including feedback from V2 to V1, as well as those involving subcortical structures, which, in turn, 
provide input to MT. While we cannot exclude the possibility that some of the cooling effects we observed result from effects via these other pathways, experiments in which feedback pathways have been inactivated have revealed relatively small and nonspecific effects (Hupé et al., 1998, 2001a,b; Luo et al., 2008). We thus think it most likely that the effects we have measured are attributable to the feedforward properties of the circuit. As new tools increase the specificity with which neural circuits can be manipulated, we anticipate that such possibilities will be addressed directly.

We believe that our findings are another example of a fundamental strategy of the visual system: to use parallel processing to extend the range over which vision is useful. Even allowing for the most sophisticated, information-rich neural coding (Abeles, 1991), individual neurons possess a very limited bandwidth. By using multiple, parallel channels, information spanning a wider range of environmental stimuli can be encoded. This strategy is first manifest at the earliest possible stage in the form of separate rod and cone photoreceptors that allow vision to operate across luminance conditions ranging from moonlight to bright sunlight. Similarly, the magnocellular and parvocellular streams allow coverage of a broader range of spatial and temporal frequencies, as well as different aspects of chromaticity. Our data suggest that a similar strategy is used within cortical pathways and provides some experimental justification of the extensive cross talk present in the cortical hierarchy. By exploiting the differences in spatial scale and tuning properties of different cortical representations, the direct and indirect pathways allow MT neurons to represent a wider range of speeds, to place stimuli within a broader context, and to integrate information concerning depth and motion.

\section{References}

Abeles M (1991) Corticonics: neural circuits of the cerebral cortex. Cambridge: Cambridge UP.

Albrecht DG (1995) Visual cortex neurons in monkey and cat: effect of contrast on the spatial and temporal phase transfer functions. Vis Neurosci 12:1191-1210.

Albright TD (1984) Direction and orientation selectivity of neurons in visual area MT of the macaque. J Neurophysiol 52:1106-1130.

Anderson JC, Martin KA (2002) Connection from cortical area V2 to MT in macaque monkey. J Comp Neurol 443:56-70.

Baumann R, van der Zwan R, Peterhans E (1997) Figure-ground segregation at contours: a neural mechanism in the visual cortex of the alert monkey. Eur J Neurosci 9:1290-1303.

Bisti S, Carmignoto G, Galli L, Maffei L (1985) Spatial-frequency characteristics of neurones of area 18 in the cat: dependence on the velocity of the visual stimulus. J Physiol 359:259-268.

Burkhalter A, Van Essen DC (1986) Processing of color, form and disparity information in visual areas VP and V2 of ventral extrastriate cortex in the macaque monkey. J Neurosci 6:2327-2351.

Carl JR, Gellman RS (1987) Human smooth pursuit: stimulus-dependent responses. J Neurophysiol 57:1446-1463.

Cavanaugh JR, Bair W, Movshon JA (2002a) Nature and interaction of signals from the receptive field center and surround in macaque V1 neurons. J Neurophysiol 88:2530-2546.

Cavanaugh JR, Bair W, Movshon JA (2002b) Selectivity and spatial distribution of signals from the receptive field surround in macaque V1 neurons. J Neurophysiol 88:2547-2556.

Churchland MM, Priebe NJ, Lisberger SG (2005) Comparison of the spatial limits on direction selectivity in visual areas MT and V1. J Neurophysiol 93:1235-1245.

DeAngelis GC, Newsome WT (1999) Organization of disparity-selective neurons in macaque area MT. J Neurosci 19:1398-1415.

DeAngelis GC, Uka T (2003) Coding of horizontal disparity and velocity by MT neurons in the alert Macaque. J Neurophysiol 89:1094-1111.

Derrington AM, Lennie P (1984) Spatial and temporal contrast sensitivities of neurones in lateral geniculate nucleus of macaque. J Physiol 357:219-240

Desimone R, Ungerleider LG (1986) Multiple visual areas in the caudal superior temporal sulcus of the macaque. J Comp Neurol 248:164-189.

DeYoe EA, Van Essen DC (1985) Segregation of efferent connections and receptive field properties in visual area V2 of the macaque. Nature 317:58-61.

Duncan RO, Albright TD, Stoner GR (2000) Occlusion and the interpretation of visual motion: perceptual and neuronal effects of context. J Neurosci 20:5885-5897.

Felleman DJ, Van Essen DC (1991) Distributed hierarchical processing in the primate cerebral cortex. Cereb Cortex 1:1-47.

Foster KH, Gaska JP, Nagler M, Pollen DA (1985) Spatial and temporal frequency selectivity of neurones in visual cortical areas Vl and V2 of the macaque monkey. J Physiol (Lond) 365:331-363.

Frazor RA, Albrecht DG, Geisler WS, Crane AM (2004) Visual cortex neurons of monkeys and cats: temporal dynamics of the spatial frequency response function. J Neurophysiol 91:2607-2627.

Gattass R, Gross CG, Sandell JH (1981) Visual topography of V2 in the macaque. J Comp Neurol 201:519-539.

Gattass R, Sousa AP, Gross CG (1988) Visuotopic organization and extent of V3 and V4 of the macaque. J Neurosci 8:1831-1845.

Groh JM, Born RT, Newsome WT (1997) How is a sensory map read out? Effects of microstimulation in visual area MT on saccades and smooth pursuit eye movements. J Neurosci 17:4312-4330.

Hicks TP, Lee BB, Vidyasagar TR (1983) The responses of cells in macaque lateral geniculate nucleus to sinusoidal gratings. J Physiol 337:183-200.

Huang X, Albright TD, Stoner GR (2008) Stimulus dependency and mechanisms of surround modulation in cortical area MT. J Neurosci 28:13889-13906.

Hubel DH, Livingstone MS (1987) Segregation of form, color, and stereopsis in primate area 18. J Neurosci 7:3378-3415.

Hupé JM, James AC, Payne BR, Lomber SG, Girard P, Bullier J (1998) Cortical feedback improves discrimination between figure and background by V1, V2 and V3 neurons. Nature 394:784-787.

Hupé JM, James AC, Girard P, Lomber SG, Payne BR, Bullier J (2001a) Feedback connections act on the early part of the responses in monkey visual cortex. J Neurophysiol 85:134-145.

Hupé JM, James AC, Girard P, Bullier J (2001b) Response modulations by static texture surround in area $\mathrm{V} 1$ of the macaque monkey do not depend on feedback connections from V2. J Neurophysiol 85:146-163.

Kapadia MK, Westheimer G, Gilbert CD (1999) Dynamics of spatial summation in primary visual cortex of alert monkeys. Proc Natl Acad Sci U S A 96:12073-12078.

Krekelberg B, van Wezel RJ, Albright TD (2006) Interactions between speed and contrast tuning in the middle temporal area: implications for the neural code for speed. J Neurosci 26:8988-8998.

Lagae L, Raiguel S, Orban GA (1993) Speed and direction selectivity of macaque middle temporal neurons. J Neurophysiol 69:19-39.

Levitt JB, Yoshioka T, Lund JS (1994a) Intrinsic cortical connections in macaque visual area V2: evidence for interaction between different functional streams. J Comp Neurol 342:551-570.

Levitt JB, Kiper DC, Movshon JA (1994b) Receptive fields and functional architecture of macaque V2. J Neurophysiol 71:2517-2542.

Lisberger SG, Movshon JA (1999) Visual motion analysis for pursuit eye movements in area MT of macaque monkeys. J Neurosci 19:2224-2246.

Lisberger SG, Westbrook LE (1985) Properties of visual inputs that initiate horizontal smooth pursuit eye movements in monkeys. J Neurosci 5:1662-1673.

Liu J, Newsome WT (2003) Functional organization of speed tuned neurons in visual area MT. J Neurophysiol 89:246-256.

Lomber SG, Payne BR, Horel JA (1999) The cryoloop: an adaptable reversible cooling deactivation method for behavioral or electrophysiological assessment of neural function. J Neurosci Methods 86:179-194.

Luo F, Wang Q, Kashani A, Yan J (2008) Corticofugal modulation of initial sound processing in the brain. J Neurosci 28:11615-11621.

Madelain L, Krauzlis RJ (2003) Pursuit of the ineffable: perceptual and motor reversals during the tracking of apparent motion. J Vis 3:642-653.

Maunsell JH, van Essen DC (1983) The connections of the middle temporal visual area (MT) and their relationship to a cortical hierarchy in the macaque monkey. J Neurosci 3:2563-2586. 
McLean J, Palmer LA (1994) Organization of simple cell responses in the three-dimensional (3-D) frequency domain. Vis Neurosci 11:295-306.

Mikami A, Newsome WT, Wurtz RH (1986) Motion selectivity in macaque visual cortex. II. Spatiotemporal range of directional interactions in MT and V1. J Neurophysiol 55:1328-1339.

Moulden B, Kingdom F, Gatley LF (1990) The standard deviation of luminance as a metric for contrast in random-dot images. Perception 19:79-101.

Movshon JA (1975) The velocity tuning of single units in cat striate cortex. J Physiol 249:445-468.

Movshon JA, Newsome WT (1996) Visual response properties of striate cortical neurons projecting to area MT in macaque monkeys. J Neurosci 16:7733-7741.

Nassi JJ, Callaway EM (2006) Multiple circuits relaying primate parallel visual pathways to the middle temporal area. J Neurosci 26:12789-12798.

Newsome WT, Wurtz RH, Dürsteler MR, Mikami A (1985) Deficits in visual motion processing following ibotenic acid lesions of the middle temporal visual area of the macaque monkey. J Neurosci 5:825-840.

Orban GA, Kennedy H, Bullier J (1986) Velocity sensitivity and direction selectivity of neurons in areas V1 and V2 of the monkey: influence of eccentricity. J Neurophysiol 56:462-480.

Pack CC, Gartland AJ, Born RT (2004) Integration of contour and terminator signals in visual area MT of alert macaque. J Neurosci 24:3268-3280.

Pack CC, Hunter JN, Born RT (2005) Contrast dependence of suppressive influences in cortical area MT of alert macaque. J Neurophysiol 93:1809-1815.

Pack CC, Conway BR, Born RT, Livingstone MS (2006) Spatiotemporal structure of nonlinear subunits in macaque visual cortex. J Neurosci 26:893-907.

Peterhans E, von der Heydt R (1993) Functional organization of area V2 in the alert macaque. Eur J Neurosci 5:509-524.

Polat U, Mizobe K, Pettet MW, Kasamatsu T, Norcia AM (1998) Collinear stimuli regulate visual responses depending on cell's contrast threshold. Nature 391:580-584.

Ponce CR, Lomber SG, Born RT (2008) Integrating motion and depth via parallel pathways. Nat Neurosci 11:216-223.

Priebe NJ, Cassanello CR, Lisberger SG (2003) The neural representation of speed in macaque area MT/V5. J Neurosci 23:5650-5661.
Priebe NJ, Lisberger SG, Movshon JA (2006) Tuning for spatiotemporal frequency and speed in directionally selective neurons of macaque striate cortex. J Neurosci 26:2941-2950.

Qiu FT, von der Heydt R (2005) Figure and ground in the visual cortex: v2 combines stereoscopic cues with gestalt rules. Neuron 47:155-166.

Raiguel SE, Xiao DK, Marcar VL, Orban GA (1999) Response latency of macaque area MT/V5 neurons and its relationship to stimulus parameters. J Neurophysiol 82:1944-1956.

Rockland KS (1995) Morphology of individual axons projecting from area V2 to MT in the macaque. J Comp Neurol 355:15-26.

Sceniak MP, Ringach DL, Hawken MJ, Shapley R (1999) Contrast's effect on spatial summation by macaque V1 neurons. Nat Neurosci 2:733-739.

Shapley RM, Victor JD (1978) The effect of contrast on the transfer properties of cat retinal ganglion cells. J Physiol 285:275-298.

Sincich LC, Horton JC (2003) Independent projection streams from macaque striate cortex to the second visual area and middle temporal area. J Neurosci 23:5684-5692.

Standage GP, Benevento LA (1983) The organization of connections between the pulvinar and visual area MT in the macaque monkey. Brain Res 262:288-294.

Tolhurst DJ, Movshon JA (1975) Spatial and temporal contrast sensitivity of striate cortical neurones. Nature 257:674-675.

Van Essen DC, Maunsell JH, Bixby JL (1981) The middle temporal visual area in the macaque: myeloarchitecture, connections, functional properties and topographic organization. J Comp Neurol 199:293-326.

Van Essen DC, Anderson CH, Felleman DJ (1992) Information processing in the primate visual system: an integrated systems perspective. Science 255:419-423.

Yabuta NH, Sawatari A, Callaway EM (2001) Two functional channels from primary visual cortex to dorsal visual cortical areas. Science 292:297-300.

Zar JH (1999) Biostatistical analysis (Ryu T, ed). Upper Saddle River, NJ: Prentice-Hall.

Zeki SM (1969) The secondary visual areas of the monkey. Brain Res 13:197-226

Zhou H, Friedman HS, von der Heydt R (2000) Coding of border ownership in monkey visual cortex. J Neurosci 20:6594-6611. 